\title{
Modulation of auditory neural responses by a visual context in human fear conditioning
}

\author{
Jorge L. Armony ${ }^{1,2, C A}$ and Raymond J. Dolan ${ }^{1,3}$
}

\begin{abstract}
'Wellcome Department of Cognitive Neurology, Institute of Neurology, 12 Queen Square, London WCIN 3BG; ${ }^{2}$ Institute of Cognitive Neuroscience, University College London, 17 Queen Square, London WCIN 3AR; ${ }^{3}$ The Royal Free Hospital School of Medicine, Rowland Street, London NW3 2PF, UK

${ }^{\mathrm{CA}, 2}$ Corresponding Author and Address
\end{abstract}

Received 8 August 200I; accepted I5 August 200I

Responses to a stimulus signaling danger depend not only on the nature of that stimulus, but also on the context in which it is presented. A large body of work has been conducted in experimental animals investigating the neural correlates of contextual modulation of fear responses. However, much less is known about this process in humans. In this study we used functional $M R I$ in a fear conditioning paradigm to explore this phenomenon. Responses to acoustic conditioned stimuli in

Key words: Emotion; fMRI; Neuroimaging; Parietal cortex auditory cortex were modulated by the presence of a visual context which signaled the likelihood of receiving an aversive unconditioned stimulus. Furthermore, the presence of the aversive visual context was associated with enhanced activity in parietal cortex, which may reflect an increase in attention to the presence of environmental threat stimuli. NeuroReport 12:3407-34 I (C) 200 I Lippincott Williams \& Wilkins.

\section{INTRODUCTION}

When a neutral stimulus (the conditioned stimulus, CS) is repeatedly presented in association with an aversive event (the unconditioned stimulus, US), it comes to elicit a wide range of species-specific defensive responses, even when presented in the absence of the US. This phenomenon, known as aversive classical conditioning or fear conditioning, is an efficient form of emotional learning through which novel environmental stimuli gain access to neural circuits that control hardwired, evolutionary acquired, defense responses [1-3]. Nonetheless, indiscriminate fear responses to a conditioned stimulus can be maladaptive; the same physical stimulus may be predictive of the occurrence of an aversive event in one context, but not in others. Thus, it is important not only to learn to detect a stimulus that signals potential threat, but also to discriminate the context within which it occurs. This latter form of learning is generally known as contextual conditioning, and has been extensively studied in animals, particularly rodents $[4,5]$.

In humans, interactions between an aversive stimulus and context have particular clinical relevance in the treatment of certain disorders, such as drug adddiction [6], post-traumatic stress disorder [7] and phobias [8]. However, although several neuroimaging studies have recently explored the neural substrates of fear conditioning in healthy humans [9], less is known about the influence of contextual cues on the expression of conditioned responses. The goal of this study was to investigate the neural correlates of the contextual modulation of fear conditioning in healthy volunteers using event-related fMRI.

During scanning, subjects were exposed to a differential auditory fear conditioning procedure: one tone (the conditioned stimulus $\mathrm{CS}+$ ) was paired with an aversive unconditioned stimulus whereas another tone of a different frequency (the CS-) was not. Critically, the CS+ was paired with the US only when the visual background was set to a particular color $(\mathrm{CTX}+)$ but not when the other color $(\mathrm{CTX}-$ ) was present (Fig. 1). Thus, the background color provided a context signal which indexed the overall likelihood of receiving a US. In other words, the presence of the CTX - color represented a safe temporal context, during which no US would be delivered, regardless of the presentation of the CSs. In contrast, the CTX+ color signaled the probability of US delivery (contingent upon the presentation of a CS+). Throughout the experiment, skin conductance responses were recorded as a measure of autonomic conditioned responses.

\section{MATERIALS AND METHODS}

Subjects: Eight healthy volunteers (three male, five female), without a history of neurological or psychiatric impairments, participated in this study. A structural MRI scan was taken to exclude subjects who showed any evidence of structural abnormalities. Subjects provided written informed consent before the experiment, and all procedures were approved by the Joint Ethics Committee of National Hospital and Institute of Neurology. 


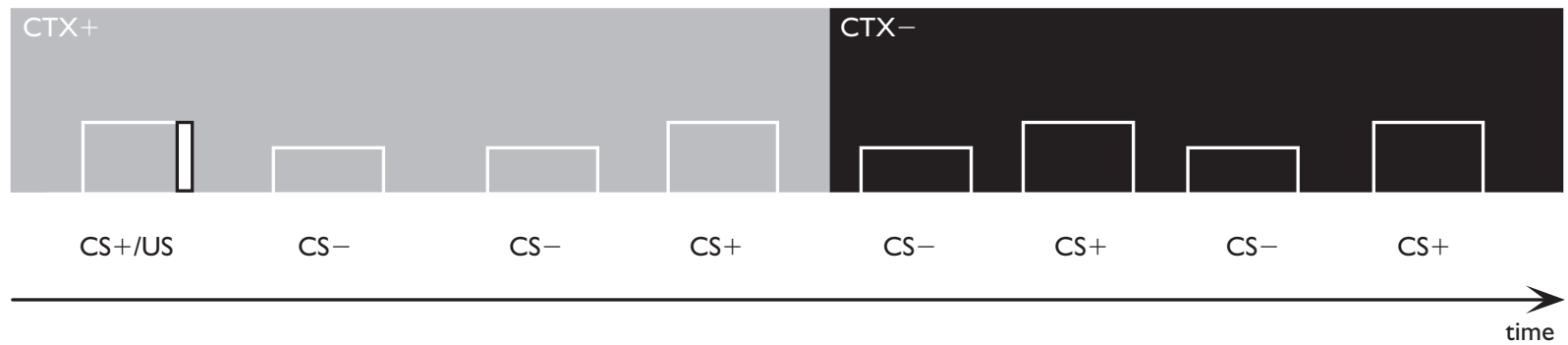

Fig. I. Schematic of the experimental design. Two tones of different frequencies served as the CS+ (paired with an aversive US), and CS-. The background color of a computer monitor served as the context. In the presence of the safe context (CTX-), no US was delivered, whereas in the conditioning context (CTX+), the CS + was paired with the US in $50 \%$ of the trials.

Experimental design: Subjects were presented with two auditory stimuli (pure tones, 440 and $5300 \mathrm{~Hz}$ ) through MRI-compatible headphones. The tones had a duration of $3 \mathrm{~s}$, an average intensity of $80 \mathrm{~dB}$ and a mean stimulus onset asynchrony (SOA) of $10 \mathrm{~s}$. For each subject, one of the tones was chosen as the CS+ and thus co-terminated with a US $(200 \mathrm{~ms}$ burst of white noise, average intensity $100 \mathrm{~dB}$ ), and the other as the CS- (never paired with the US). Throughout the experiment, subjects were looking, through a mirror mounted on the headcoil, at a computer monitor in order to perform a target discrimination task (see below).

The background screen color alternated between two colors, green and blue (average duration $60 \mathrm{~s}$ ). The screen color provided the conditioning context. When one of the colors was present $(\mathrm{CTX}+)$, the $\mathrm{CS}+$ was paired with the US, but when the other color was on, the US was never delivered. Thus, the background color provided a context signal which indicated the level of overall likelihood of receiving a US. In other words, the presence of the CTXcolor represented a safe temporal context, during which no US would be delivered, regardless of the presentation of the CSs. In contrast, the CTX + color signaled the probability of US delivery (contingent upon the presence of a $\mathrm{CS}+$ ). A schematic of the design is shown in Fig. 1.

We used a $50 \%$ partial reinforcement schedule (i.e. only half of the presentations of the CS+ were paired with the US) to allow us to investigate the haemodynamic response to the CS+ uncontaminated by the US $[10,11]$. Thus, in the ensuing analysis and results, $\mathrm{CS}+$ refers to the presentation of the CS+ in the absence of the US (note that in the CTXcondition, the CS+ was always presented alone, without a US). The assignment of tones as CS + and colors as CTX+ was counterbalanced across subjects. Subjects were told that sounds would be played during the experiment, but that these were unrelated to their task and merely served as distracting stimuli. All subjects were debriefed after scanning.

Behavioral measurements: Skin conductance responses (SCRs) were monitored throughout the experiment and used as an index of autonomic conditioning. SCRs were measured using $\mathrm{Ag} / \mathrm{AgCl}$ electrodes attached to the palmar surface of the middle phalanges of the index and middle fingers. SCR data was analysed as described previously $[10,11]$. Briefly, data were digitized, detrended, temporally smoothed to remove MRI scanning artifacts, and resampled at $10 \mathrm{~Hz}$. SCR amplitudes for each tone presentation were calculated as the maximal deflection from baseline. Values were square-root transformed to attain statistical normality [12] and entered in a $2 \times 2$ $(\mathrm{CS} \times \mathrm{CTX})$ repeated measures ANOVA.

During the scanning session, subjects performed a visual discrimination task, consisting in identifying, as quickly and accurately as possible, whether a peripheral $\mathrm{T}$ stimulus appearing on either side of the visual field was up or down, by pressing one of two buttons in a keypad. The presentation of the visual stimuli coincided with the offset of the auditory stimuli. This allowed us to use reaction times as another behavioural measure of conditioning. That is, we hypothesized that the expectation of an aversive US, associated with the CS+, would interfere with the response to the visual target, resulting in longer reaction times.

MRI scanning: Images were acquired with a $2 \mathrm{~T}$ Magnetom VISION whole-body MRI system (Siemens, Erlangen, Germany) equipped with a head volume coil. T2*weighted echoplanar image volumes with blood oxygenation level-dependent (BOLD) contrast (echo time, $40 \mathrm{~ms}$; $64 \times 64$ pixels) were acquired in an axial orientation. Each volume comprised of 32 slices (slice thickness $3 \mathrm{~mm}$ ), positioned to cover the whole brain. The effective repetition time (TR) was $3.2 \mathrm{~s} / \mathrm{vol}$. To minimize head motion, subjects were restrained with bitemporal pressure pads. A total of 600 volumes were acquired for each subject, over $30 \mathrm{~min}$.

Data analysis: Image processing and statistical analysis were performed using a fixed-effects model in SPM99. The SPM methodology is described in detail elsewhere $[13,14]$. Briefly, the imaging time series was realigned to the first volume, normalized to a standard Talairach space, and spatially smoothed.

Data were analyzed by modeling the evoked haemodynamic responses for the different stimuli as delta functions, locked to the onset of the tones and the changes in background color, convolved with a synthetic haemodynamic function and its temporal derivative. We defined seven event types: CTX+ onset, CTX- onset, CS+/ $\mathrm{US}(\mathrm{CTX}+), \mathrm{CS}+(\mathrm{CTX}+), \mathrm{CS}-(\mathrm{CTX}+)$, and $\mathrm{CS}+(\mathrm{CTX}-)$ and CS-(CTX-) (Fig. 1). We also included the six movement parameters obtained from the realignment procedure as confounds to minimize the chances of detecting false activations due to movement artifacts. Differential effects 
were tested by applying appropriate linear contrasts to the parameter estimates of each event type, resulting in a tstatistic for each voxel. These t-statistics (transformed to Zstatistics) constitute a statistical parametric map (SPM). The corresponding $p$-values were corrected for multiple comparisons across the entire brain, except where otherwise indicated.

Previous fMRI studies of fear conditioning have shown that CS-elicited responses in some brain regions, particularly in the amygdala, decrease over time and thus are best characterized by time $\times$ event interactions $[10,11]$. We therefore defined, in addition to the design described above, new regressors representing the time $\times$ condition interactions for each event type. These additional regressor were created by multiplying the original regressors with a mean corrected exponentially decaying function with a time constant of one-quarter of the scanning session length.

\section{RESULTS}

Skin conductance responses to the two auditory stimuli $\left(\mathrm{CS}+\right.$ and $\left.\mathrm{CS}_{-}\right)$in each context $(\mathrm{CTX}+$ and $\mathrm{CTX}-)$ were submitted to a repeated measures, $2 \times 2$ factorial ANOVA. This analysis yielded a significant main effect of context $(F(7,1)=7.11, p<0.05)$, but no significant main effect of CS $(\mathrm{F}<1)$ or interaction between context and CSs $(\mathrm{F}<1)$. SCRs were larger for both the CS+ and CS - in the CTX+ than in the CTX- (Fig. 2). These results suggest that subjects learned the difference in the predictive value of the two contexts.

A similar ANOVA analysis of the reaction times to target discrimination yielded a trend of a main effect of CS $(\mathrm{F}(7,1)=4.36, p=0.07)$, and no effect of context or interaction. This comparison achieved statistical significance when restricted to a comparison between $\mathrm{CS}+$ and $\mathrm{CS}$ in the CTX $+(\mathrm{t}(7)=2.68, p<0.05)$.

We next examined the neural correlates of the difference in autonomic responses to the conditioned stimuli as a function of context by contrasting the auditory-evoked haemodynamic responses to both the $\mathrm{CS}+$ and $\mathrm{CS}-$ in the two contexts. That is, we looked for regions which responded differently to the CSs depending on the context in which they were presented (i.e. main effect of context on auditory responses). To avoid potential confounding effects of the US presentation, we only included in the analysis those presentations of the CS+ that were not paired with the US. This comparison revealed a significant bilateral activation of auditory cortex (Right: $\mathrm{x}=62, \mathrm{y}=-12, \mathrm{z}=8$, $p<0.01$; Left: $\mathrm{x}=-56, \mathrm{y}=-18, \mathrm{z}=12, p<0.002$; Fig. 3a). As shown in Fig. $3 b$, the auditory cortex responses were larger to acoustic stimuli in the safe context (CTX-) than in the conditioning context $(\mathrm{CTX}+)$. This result demonstrates that the same auditory stimuli elicited different responses in auditory cortex depending on the visual context (background color).

To examine which areas were involved in the distinction between the contexts themselves, we compared the sustained responses during the entire duration of CTX+ versus CTX-. This contrast revealed significant activation of parietal cortex in the right hemisphere $(x=58, y=-48$, $\mathrm{z}=22, p<0.05$, corrected for multiple comparisons) and, to a lesser degree, in the corresponding area in the left hemisphere $(\mathrm{x}=-50, \mathrm{y}=-38, \mathrm{z}=34, p<0.001$ uncorrected). The location of these activations, superimposed onto a standard T1 template is shown in Fig. 3c, and the corresponding peristimulus-time histograms are depicted in Fig. 3c.

Finally, we tested for regions responding to the $\mathrm{CS} \times \mathrm{CTX}$ interaction. That is, we performed the contrast $\left(\mathrm{CS}+-\mathrm{CS}_{-}\right)$in $\mathrm{CTX}+$ ws $\left(\mathrm{CS}+-\mathrm{CS}_{-}\right)$in $\mathrm{CTX}-$, as a function of scanning time (see Materials and Methods). We observed significant posterior right amygdala activation ( $\mathrm{x}=30, \mathrm{y}=-10, \mathrm{z}=-8 ; p<0.001$ uncorrected $)$.

It is important to emphasize that, as described in Materials and Methods, the results reported here were obtained from a fixed-effects analysis, which limits the generalizability of the present findings. Nonetheless, all the (a)

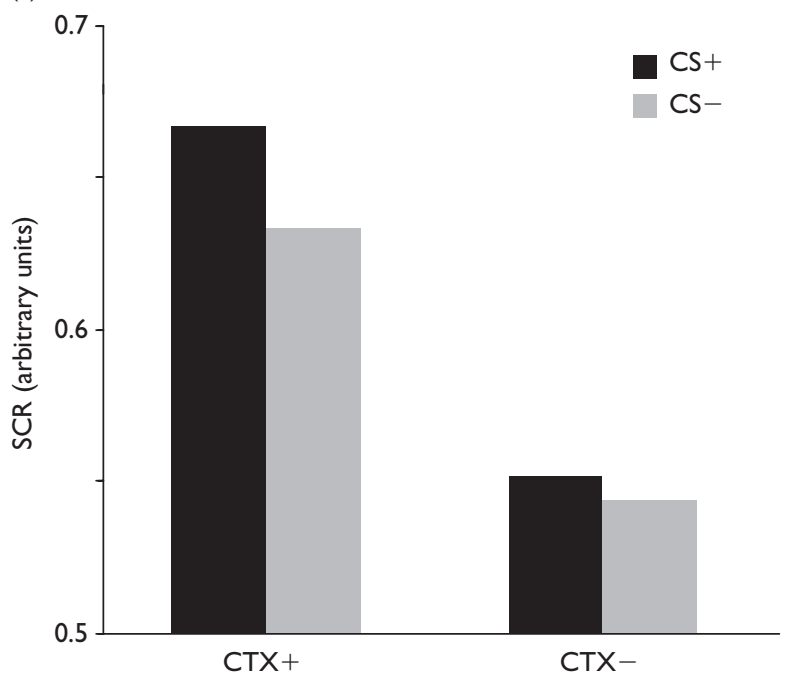

(b)

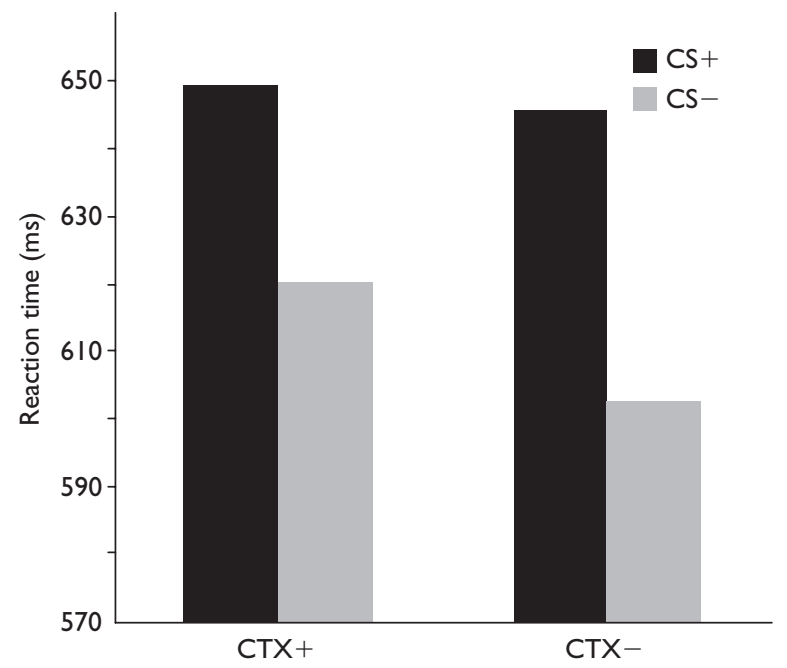

Fig. 2. (a) Reaction times in the visual discrimination task and (b) skin conductance responses (SCR) to the CS+ and CS-, as a function of conditioned stimulus and context. 
(a)
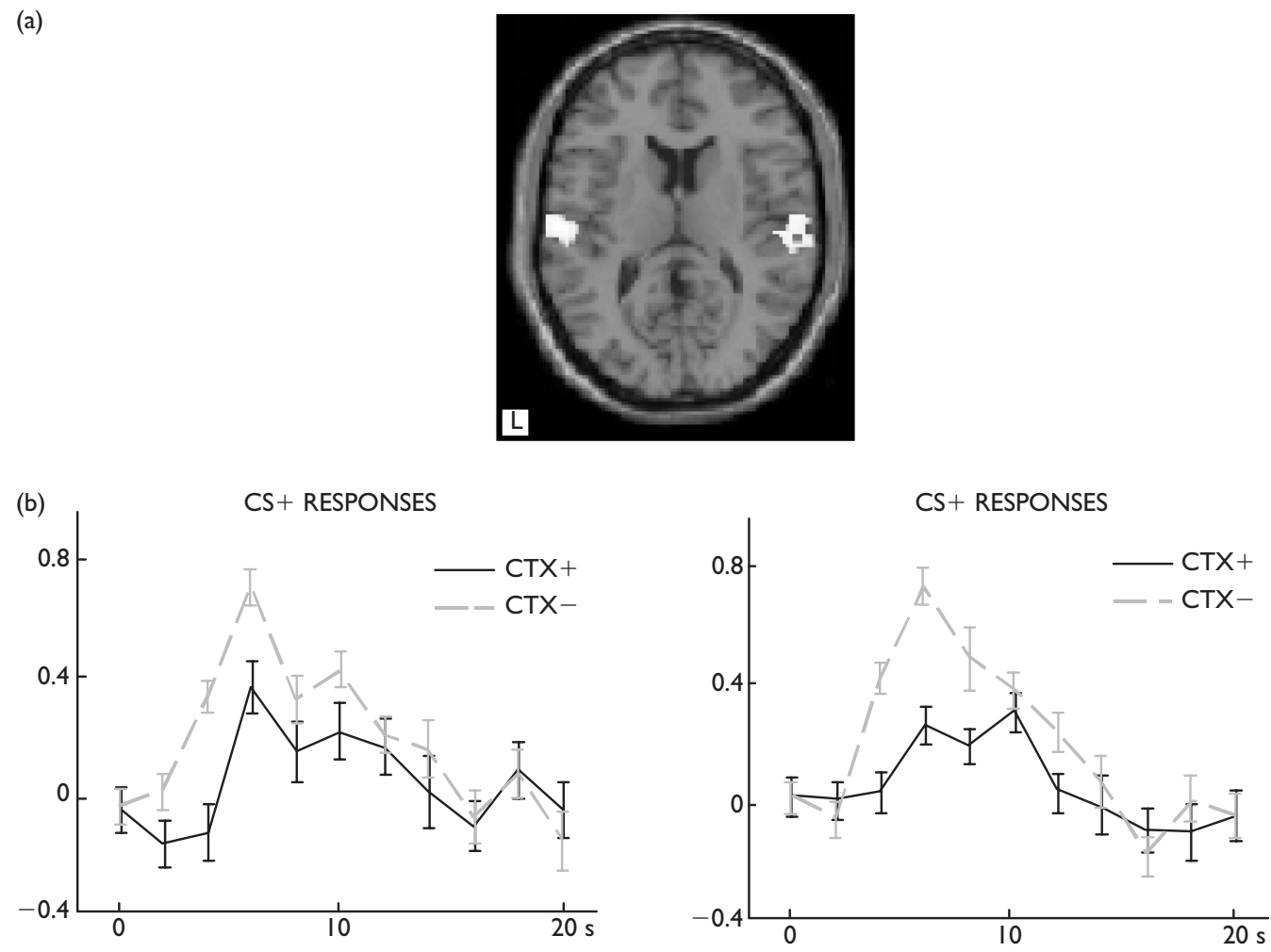

(c)

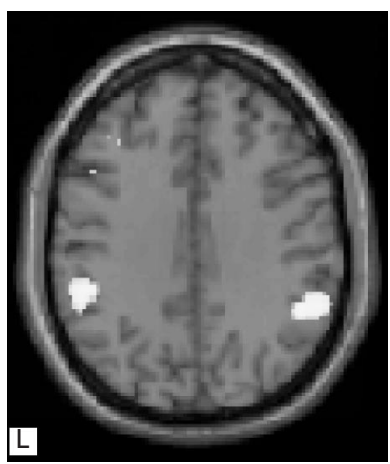

(d)

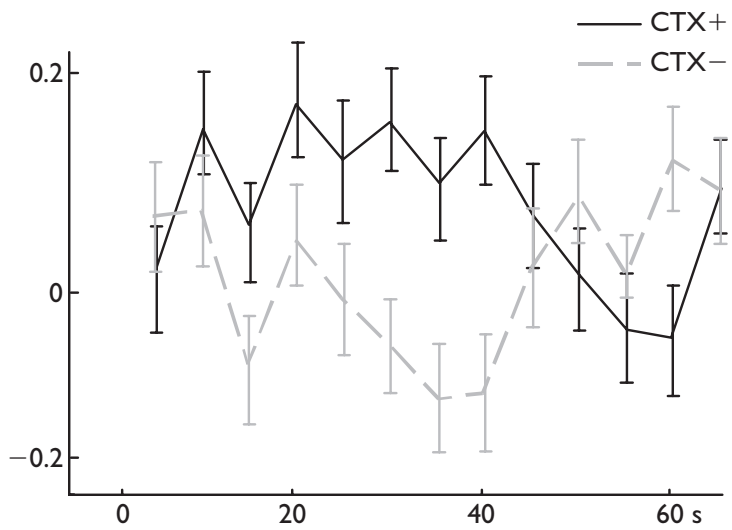

Fig. 3. (a) Activation of auditory cortex in response to the CS+ and CS- in CTX-, compared to the responses to the same stimuli in CTX+. (b) Group-averaged peristimulus time histograms of right auditory cortex responses to CS+ (left) and CS - (right) in CTX+ and CTX-. (c) Activation of parietal cortex during the presence of the CTX+, compared to the CTX + . (d) Group-averaged peristimulus time histograms of right parietal cortex responses to $\mathrm{CTX}+$ and $\mathrm{CTX}-$.

activations reported here showed to be significant, in a post-hoc random effects analysis, with $p$ values between 0.01 and 0.0005 , suggesting a reasonable amount of consistency of the effects across subjects.

\section{DISCUSSION}

Our findings show that responses to conditioned stimuli in auditory cortex can be modulated by the context in which they are presented. That is, the same physical stimuli produced greater activation in the context in which the US had not been presented (safe context) than in the condition- ing context. This result is consistent with previous imaging findings by Morris and colleagues [15] showing decreased activation in auditory cortex for the CS+, compared to the $\mathrm{CS}-$, in a standard discriminatory fear conditioning paradigm. These findings in auditory cortex may be related not to the modality of the CS but rather to that of the US (a loud burst of white noise in both studies). For example, similar decreased responses to a CS were observed by Wik et al. [16] in somatosensory cortex, using MEG, in anticipation of a shock US. The authors suggested that decreases in sensory cortex in response to a CS may reflect an antici- 
patory response that serves to reduce the noxious effects of the US. Consistent with this idea, the stimuli producing a decreased auditory response in the Morris et al. [15] study and ours were those associated with enhanced anticipatory skin conductance responses, which are part of the human repertoire of autonomic defense responses elicited in the presence of a potentially dangerous experience.

Similarly, the time-dependent differential amygdala activation to the $\mathrm{CS}+$ in the CTX+ is in agreement with a large literature supporting its role in fear conditioning $[1,3,17]$ and, in particular, with previous imaging studies (for a review see [9]). This activation, specific for the CS+ in the CTX+, appears to be inconsistent with the SCR results described above, which showed enhanced responses to both $\mathrm{CS}+$ and CS - in the CTX + . This discrepancy could be due to the variability of SCR as a measure of conditioning and the relatively small number of subject tested in this study, as there seems to be a non-significant trend for larger SCRs to the CS+ (see Fig. 2). However, our other behavioural measure of conditioning, reaction times, did reveal a significantly larger value for the $\mathrm{CS}+$ trials in the $\mathrm{CTX}+$ when compared to the CS-. Nonetheless, further studies are necessary to fully elucidate the relationship between amygdala activation as revealed by fMRI and the various behavioral and physiological measures of conditioning.

Finally, the increased activation of parietal cortex during the presence of the contextual cue associated with the delivery of the US is consistent with an enhanced attention to the environment during that time, in order to monitor the probable delivery of an aversive stimulus. Posterior parietal cortex has been consistently implicated in selective attention processes $[18,19]$. The observed differential activation of right parietal cortex is also in agreement with the hypothesis that this area plays a role in modulating behavioral and autonomic arousal in response to emotional information [20].

The lack of hippocampal activation in this experiment may appear somewhat surprising, given that several lesion studies in experimental animals have consistently supported the notion that this brain region plays a key role in contextual conditioning $[5,21,22]$. It is important to point out that in most of those studies, the different contexts usually were physically different environments, each providing a variety of distinctive multimodal cues. In contrast, using a single-modality context (background sound), Rawlins et al. [23] did not find an effect of hippocampal lesions on the contextual modulation of conditioned responses to a visual CS. Thus, it appears that the hippocampus may not necessarily be involved in the contextual modulation of conditioned responses per se, but rather in the integration of different multimodal cues of different physical nature to provide a more complex stimulus representation of the environment. This hypothesis is consistent with a number of theories of hippocampal spatial, configural and relational processing [24-26]. Naturally, it is also possible that our lack of significant hippocampal activation reflects a
Type II error, a possibility which remains open to future investigation.

\section{CONCLUSION}

We have shown that, in humans, the affective value of a contextual cue can modulate autonomic and neural responses to a conditioned stimulus. Specifically, the presentation of an auditory stimulus within a safe context (one that had not been associated with aversive events) elicited a stronger activation in auditory cortex, compared to the same stimulus presented in an aversive context. In turn, the presence of the conditioned context was associated with enhanced activation within parietal cortex. These results suggest that processing of information in primary sensory areas is modulated by the affective value of the context in which they are presented, possibly by way of inputs from higher order areas, such as the parietal cortex. This modulatory system may become maladaptive in some individuals, leading to a variety of emotional disorders, such as post-traumatic stress syndrome and certain phobias.

\section{REFERENCES}

1. Fanselow MS. Psychonom Bull Rev 1, 429-438 (1994).

2. Kapp BS, Whalen PJ, Supple WF et al. Amygdaloid contributions to conditioned arousal and sensory information processing. In: Aggleton JP, ed. The Amygdala: Neurobiological Aspects of Emotion, Memory, and Mental Dysfunction. New York: Wiley-Liss; 1992, pp. 229-254.

3. LeDoux JE. Annu Rev Neurosci 23, 155-184 (2000).

4. Bouton ME. Psychol Bull 114, 80-99 (1993).

5. Holland PC and Bouton ME. Curr Opin Neurobiol 9, 195-202 (1999).

6. Powell J. Addiction 90, 1089-1095 (1995).

7. Grillon C, Morgan CA, 3rd, Davis M et al. Biol Psychiatry 44, 1027-1036 (1998).

8. Mineka S, Mystkowski JL, Hladek D et al. J Consult Clin Psychol 67, 599-604 (1999).

9. Büchel C and Dolan RJ. Curr Opin Neurobiol 10, 219-223 (2000)

10. Büchel C, Morris J, Dolan RJ et al. Neuron 20, 947-957 (1998).

11. Büchel C, Dolan RJ, Armony JL et al. J Neurosci 19, 10869-10876 (1999).

12. Levey AB. Measurement units in psychophysiology. In: Martin I and Venables PH (eds). Techniques in Psychophysiology. New York: Wiley; 1980, pp. 597-628.

13. Friston KJ. http://www.fil.ion.ucl.ac.uk/spm/.

14. Worsley KJ and Friston KJ. Neuroimage 2, 173-181 (1995).

15. Morris JS, Friston KJ and Dolan RJ. Proc R Soc Lond B Biol Sci 265 649-657 (1998).

16. Wik G, Elbert T, Fredrikson M et al. Neuroreport 7, 737-740 (1996).

17. Davis M. The role of the amygdala in conditioned fear. In: Aggleton JP, ed. The Amygdala: Neurobiological Aspects of Emotion, Memory, and Mental Dysfunction. New York: Wiley; 1992, 255-306.

18. Cabeza R and Nyberg L. J Cogn Neurosci 12, 1-47 (2000).

19. Mesulam MM. Ann Neurol 28, 597-613 (1990).

20. Heller W. Neuropsychology 7, 476-489 (1993).

21. Kim JJ and Fanselow MS. Science 256, 675-677 (1992).

22. Phillips RG and LeDoux JE. Behav Neurosci 106, 274-285 (1992).

23. Rawlins JN and Tanner J. Behav Brain Res 91, 61-72 (1998).

24. Cohen NJ and Eichenbaum H. Memory, Amnesia, and the Hippocampal System. Cambridge, MA: MIT Press; 1993.

25. O'Keefe J and Nadel L. The Hippocampus as a Cognitive Map. Oxford: Clarendon Press; 1978.

26. Sutherland RJ and Rudy JW. Psychobiology 17, 129-144 (1989).

Acknowledgements: We are grateful to John Morris and Christian Büchel for invaluable help, and to Patrik Vuilleumier for critical comments on an earlier version of this manuscript. This work was supported by a program grant to R.J.D. from the Wellcome Trust. J.L.A. is supported by the Brain Research Trust. 